\title{
The Relationship Between Blood Pressure Variability and Cardiovascular Risk Factors in Patients with Primary Hypertension
}

\author{
Magdás Annamária ${ }^{1}$, Benedek I $^{*}$, Belényi Boglárka ${ }^{3}$, Carasca C ${ }^{4}$, Gábos Gabriella ${ }^{5}$, Incze $A^{1}$ \\ 1 University of Medicine and Pharmacy Tîrgu Mureș - Doctoral School, Department of Internal Medicine IV \\ 2 County Clinical Emergency Hospital Tîrgu Mures, Clinic of Hematology \\ 3 University of Medicine and Pharmacy Tîrgu Mureș, Department of Internal Medicine I \\ 4 University of Medicine and Pharmacy Tîrgu Mureș, Department for Forensics Medicine \\ ${ }^{5}$ County Clinical Hospital, Department for Gastroenterology
}

\begin{abstract}
Objective: The aim of the study is to assess 24-hour blood pressure variability, circadian blood pressure profile and its relation to 24-hour blood pressure and cardiovascular risk factors in primary hypertension without any associated disease versus associated with a disease such as diabetes or chronic kidney disease. Methods: This observational study included 90 hypertensive patients, 49 with primary hypertension without associated disease and 41 patients with primary hypertension and diabetes or chronic kidney disease. Circadian blood pressure profile and 24-hour variability were assessed using ambulatory monitoring. Laboratory data regarding cardiovascular risk factors and demographic data were collected in a questionnaire. Results: The number of dipper patients was higher in the group without associated disease, but the difference was not statistically significant ( $p=0.27$ ). In both groups a positive correlation was found between 24-hour systolic blood pressure variability and 24 -hour systolic blood pressure $(p=0.029)$ and was related to age $(p=0.031)$. In the second group, systolic variability showed a positive correlation with serum triglycerides ( $p=0.006, r=0.416, \mathrm{Cl}: 0.1252$ to 0.6422). Conclusion: Our findings suggest that systolic blood pressure variability is related to age, systolic blood pressure values and serum lipid levels. To prevent end organ damage in hypertension, the assessment of ambulatory monitoring derived 24-hour systolic blood pressure variability and its reduction may be at least as important as blood pressure lowering.
\end{abstract}

Keywords: blood pressure variability, cardiovascular risk factors, ambulatory monitoring

Received: 27 October 2014 / Accepted: 20 January 2015

\section{Introduction}

Hypertension is a major cardiovascular risk factor, characterized by short-term fluctuations over 24-hour period [1]. In healthy subjects blood pressure (BP) displays a circadian pattern, with a nocturnal fall $>10 \%$ compared to daytime values, known also as dipping phenomenon. This pattern can be blunted in several metabolic and cardiovascular changes and also in secondary forms of hypertension [2]. It is well known that the incidence of cardiovascular events such as stroke or myocardial infarction is increased between 6 and 9 am as a result of increased morning blood pressure surge [3]. The consequences of hypertension depend not only on casual BP measurements, but also on BP variability [1]. Therefore, to optimize blood pressure management, to assess circadian blood pressure profile, BP variability as well as abnormal nocturnal $\mathrm{BP}$ fall and excessive morning surge, the use of 24-hour ambulatory blood pressure monitoring (ABPM) is of crucial importance. Excessive BP fluctuations occurring over 24-hour period have been associated with subclinical target organ damage $[4,5]$. The latest studies reveal that BP variability lowering over 24-hour is at least as important as BP lowering [6,7]. Therefore, the new Guidelines for the management of arte-

* Correspondence to: István Benedek

E-mail: benedekistvan73@yahoo.com rial hypertension added to the diagnostic evaluation of the hypertensive patients the assessment of blood pressure variability [8]. Different methods are used to determine shortterm (within 24-hour) BP variability [1]. In our study we aimed to determine blood pressure variability using a recently proposed index, the average real variability (ARV), which allows a more accurate assessment of the circadian BP profile than the commonly used standard deviation [9]. The aim of the study is to assess 24-hour blood pressure variability, morning BP surge and its relation to 24-hour blood pressure, and cardiovascular risk factors in primary hypertension without associated disease versus associated with a disease such as diabetes or chronic kidney disease.

\section{Methods}

This observational study included 90 hypertensive patients, who were admitted to County Hospital- Department of Internal Medicine IV, Tîrgu-Mureş, between 2012-2014. All subjects gave written informed consent, and the study was approved by local Ethical Committee according to the International Ethical Guidelines and Declaration of Helsinki. Patients were divided in two groups: group 1 included 49 patients with primary hypertension without associated disease, group 2 included 41 patients with primary hypertension and diabetes or chronic kidney disease. All patients have history of hypertension, office blood pres- 
sure $>140 / 90 \mathrm{mmHg}$. Inclusion criteria were: history of hypertension, ABPM criteria: mean $\mathrm{BP}>130 / 80 \mathrm{mmHg}$, diabetes mellitus type 2, chronic kidney disease. Pregnant women, patients with type 1 diabetes mellitus, angina pectoris, congestive heart failure, arrhythmias, significant valvular disease were excluded from the study.To assess circadian blood pressure profile, 24-hour ABPM measurement was performed with a validated device (Meditech, ABPM $05)$. The daily monitoring begun between 8:00-10:00 a.m. Blood pressure was measured automatically every 15 minutes at daytime (06:00-21:59) and nighttime (22:0005:59). Blood pressure data (awake, asleep and 24-hour mean BP, awake/asleep ratio, pulse pressure, BP surge) were edited automatically. Criteria for valid measurements were: at least 48 blood pressure values, successful measurement ratio $>70 \%$. Blood pressure variability was calculated according to the formula of average real variability with a computerized program [10].

Laboratory data reflecting cardiovascular risk factors (total cholesterol, triglycerides, blood sugar, glomerular filtration rate (appreciated by Modification of Diet in Renal Disease) as well as demographic data were included in a questionnaire.

Data were collected as raw data, using MS Excel program, and statistical analysis were performed by Graph Pad Prism version 6 statistical software. Numerical data are represented as mean $\pm S D$. Means were compared using t-test for continuous variables and Chi-square test for categorical variables. Correlations were calculated with Pearson's correlation test for data representing Gaussian distribution. A $\mathrm{p}$ value $\leq 0.05$ was considered statistically significant with a confidence interval was set at $95 \%$.

\section{Results}

In the first group 49 patients (19 male, 30 female, mean age $61.92 \pm 1.77$ years) with essential hypertension were included, while in the second group (with essential hypertension and associated disease) 41 patients (19 male, 22 female, mean age $61.63 \pm 1.95$ years, $p>0.05$ ). Although in the first group $40,81 \%$ and in the second group only $29,26 \%$, of the patients were dippers, this difference was not statistically significant $(\mathrm{p}=0.27)$, as shown in Figure 1 . We found no differences between the groups regarding mean systolic/diastolic blood pressure, blood pressure variability, body mass index, total cholesterol, triglycerides, glomerular filtration rate $(\mathrm{p}>0.05)$. Morning blood pressure surge displays statistically significant difference between the groups, with much higher values in the second group, which associated diabetes or chronic kidney disease $(\mathrm{p}=0.012)$, Figure 2 . In both groups we found a positive correlation between 24-hour systolic blood pressure variability and 24-hour systolic blood pressure $(\mathrm{p}=0.029, \mathrm{r}=0.3120, \mathrm{CI}: 0.03369$ to 0.5454 in group 1 , versus $\mathrm{p}=0.01, \mathrm{r}=0.397$, CI: 0.1018 to 0.6281 in group 2 ) (Figure 3). Correlation between 24-hour diastolic blood pressure variability and 24-hour diastolic blood pressure

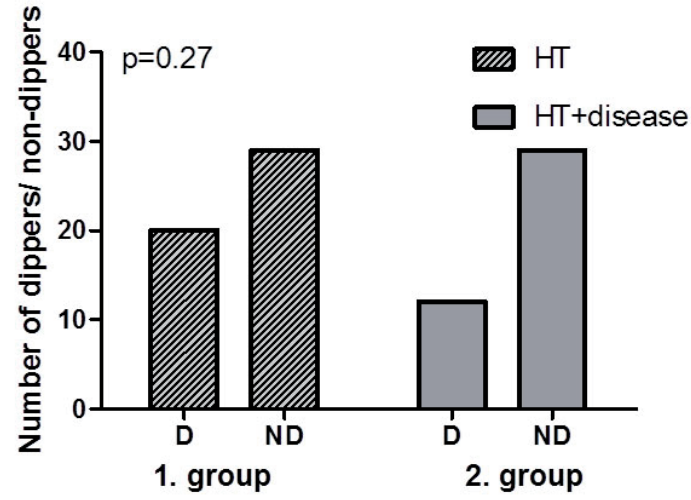

Fig. 1. Dippers/non-dippers in the study group

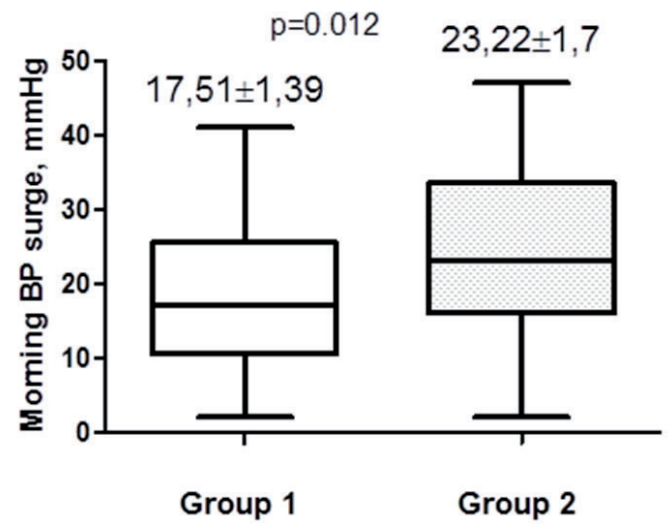

Fig. 2. Differences in morning surge between the groups

was found only in the group with essential hypertension without associated disease ( $\mathrm{p}=0.011, \mathrm{r}=0.3601$, CI: 0.8774 to 0.5824$)$. In the group with associated disease no correlation was found regarding diastolic blood pressure variability and mean blood pressure values. In group 1 , mean systolic variability was positively correlated to serum total cholesterol ( $\mathrm{p}=0.006, \mathrm{r}=0.3824$, CI: 0.1133 to 0.5992 ). In group 2 , mean systolic variability showed positive correlation with serum triglycerides $(\mathrm{p}=0.006, \mathrm{r}=0.416, \mathrm{CI}$ : 0.1252 to 0.6422 ). No correlation was found between morning blood pressure surge and 24-hour systolic/diastolic blood pressure variability $(p>0.05)$. In both of the groups we found relationship between the 24-hour systolic

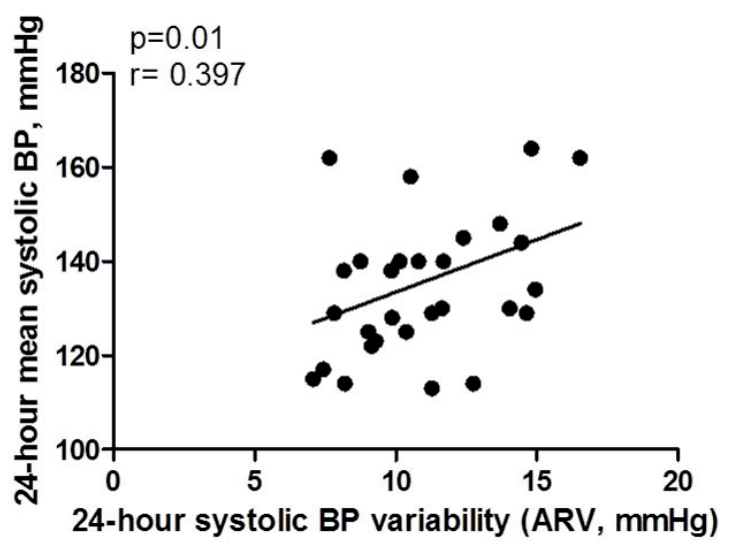

Fig. 3. Correlation between 24-hour systolic BP variability and systolic blood pressure in the group with associated disease 
blood pressure variability and age $(\mathrm{p}=0.031, \mathrm{r}=0.3071, \mathrm{CI}$ : 0.02832 to 0.5416 in group 1 , versus $\mathrm{p}=0.016, \mathrm{r}=0.3722$, CI: 0.07285 to 0.6101 in group 2), Figure 4 . In the group with hypertension and associated disease, mean diastolic blood pressure variability was associated with body mass index ( $\mathrm{p}=0.023, \mathrm{r}=0.3536, \mathrm{CI}$ : 0.005147 to 0.05964$)$.

\section{Discussion}

So far, beside the lowering of mean systolic/diastolic BP values, achievement of a dipper profile was required to prevent cardiovascular complications of hypertension. Recent studies encourage assessment and lowering of the excessive blood pressure fluctuations occurring over 24- hour $[1,11,12]$ in order to prevent end organ damage and cardiovascular complications. According to other studies, we found that in patients with essential hypertension associating diabetes or chronic kidney disease, non-dipper profile is more common, compared to subjects without associated disease [13]. Although the difference between the groups was not statistically significant, attention must be paid to normalize the dipper profile in order to prevent further decline in renal function. We found that patients with higher cardiovascular risk, presenting associated diabetes or kidney disease have greater morning blood pressure surge, which is considered a marker of cardiovascular events. These patients should benefit from a more rigorous hypertension management in order to reduce cardiovascular mortality [14]. In essential hypertension the 24-hour systolic/diastolic blood pressure variability correlates with mean systolic/diastolic blood pressure. In the first group, systolic variability was associated with total serum cholesterol. In the second group diastolic variability showed relationship with body mass index. In both groups, mean systolic variability was increased with age. According to other studies, our findings suggest that systolic blood pressure variability is associated with other cardiovascular risk factors like hypertension, age, serum total cholesterol [11]. The novelty of the present study consists in the use of the ambulatory monitoring derived short-term blood pressure variability determined by a new variability index. Most articles investigating the role of blood pressure variability in the management of hypertension, focus on visit-to visit variability, which may be not so accurate to appreciate long- term prognosis, because it may not reflect circadian blood pressure variation $(11,15,16)$.

\section{Conclusion}

In order to improve management of hypertension, ambulatory monitoring of blood pressure plays a crucial role in term of correct diagnosis. Blood pressure variability is related to age, systolic blood pressure values and serum cholesterol levels. To reduce cardiovascular risk in hypertensive patients, dipper patterns need to be achieved, ex- cessive morning blood pressure surge should be decreased and excessive blood pressure variability should be avoided.

To prevent end organ damage in hypertension, the assessment of ambulatory monitoring derived 24-hour systolic blood pressure variability and its reduction may be at least as important as blood pressure lowering. Evaluation of blood pressure variability could have an additive value in determining individual cardiovascular risk stratification. Further studies have to be conducted to identify drug classes which efficiently reduce short-term variability and normalize dipper pattern.

\section{Acknowledgment}

This paper is supported by the Sectoral Operational Programme Human Resources Development (SOP HRD), financed from the European Social Fund and by the Romanian Government under the contract number POSDRU/159/1.5/S/133377. The authors declare no conflict of interest.

\section{References}

1. Parati G, Ochoa EO, Lombardi C, Bilo G - Assessment and management of blood-pressure variability. Nature Rev Cardiol, 2013;10:143-155.

2. Mahabala C, Kamath P, Bhaskaran N et al - Antihypertensive therapy: nocturnal dippers and nondippers. Do we treat them differently? Vasc Health Risk Manag, 2013;9:125-133.

3. Tutal E, Sayin B, Ertugrul DT, et al - Is there a link between hyperuricemia, morning blood pressure surge, and non-dipping blood pressure pattern in metabolic syndrome patients? In J Nephrol Renovasc Dis, 2013;6:71-77.

4. Ciobanu AO, Gherghinescu CL, Dulgheru R, et al - The impact of blood pressure variability on subclinical ventricular, renal and vascular dysfunction, in patients with hypertension and diabetes, Maedica (Buchar), 2013;8:129-136.

5. Ozkayar N, Altun B, Yildirim T, et al - Blood pressure measurements, blood pressure variability and endothelial function in renal transplant recipients, Clin. Exp. Hypertens, online 2013. doi:10.3109/10641963.2013.827706.

6. Schmieder RE, Lehmann MV, Schmidt S - Optimizing blood pressure control in hypertension: the need to use ABPM. Blood Press, 2013;22:6572.

7. Chowdhury EK, Owen A, Krum H et al - Systolic blood pressure variability is an important predictor of cardiovascular outcomes in elderly hypertensive patients. J Hypertens, 2014;32:525-533.

8. Mancia et al - The Task Force for the management of arterial hypertension of the European Society of Hypertension (ESH) and of the European Society of Cardiology (ESC). J Hypertens, 2013;31:1281-1357.

9. Mena LJ, Maestre GE, Hansen TW et al - How many measurements are needed to estimate lood pressure variability without loss of prognostic information? Am J Hypertens, 2014;27: 46-55.

10. Mena L, Pintos S,Queipo NV et al - A reliable index for the prognostic significance of blood pressure variability. J Hypertens, 2005;23:505-511.

11. Blacher J, Safar ME, Ly C et al - Blood pressure variability: cardiovascular risk integrator or independenr risk factor? J Hum Hypertens 2014, doi:10.1038/jhh.2014.44

12. Mancia G - Short- and long-term blood pressure variability: Present and future, Hypertens 2012;60:512-517.

13. Stow LR, Gumz ML - The circadian clock in the kidney. J Am SocNephrol, 2011;22:598-604.

14. Martin CA, Cameron JD, Head GA et al - The morning blood pressure surge is related to serum cholesterol. J Hum Hypertens, 2013;27:315320

15. Mallamaci F, Tripepi G - Blood pressure variability in chronic kidney disease patients. Blood Purif, 2013;36:58-62.

16. Kawai $\mathrm{T}$ et al - The impact of visit-to-visit variability in blood pressure on renal function. Hypertens Res, 2012;35:239-243. 